\title{
Targeted delivery to inflammatory monocytes for efficient RNAi-mediated immuno-intervention in auto-immune arthritis
}

\author{
Jessy Présumey ${ }^{1,2 \dagger}$, Gabriel Courties ${ }^{1,2 \dagger}$, Louis-Marie Charbonnier ${ }^{1,2}$, Virginie Escriou ${ }^{3,4,5,6}$, Daniel Scherman ${ }^{3,4,5,6}$, \\ Yves-Marie Pers $^{1,2,7}$, Pascale Louis-Plence ${ }^{1,2}$, Diego Kyburz ${ }^{8}$, Steffen Gay ${ }^{8}$, Christian Jorgensen ${ }^{1,2,7}$, \\ Florence Apparailly ${ }^{1,2,7^{*}}$
}

From 6th European Workshop on Immune-Mediated Inflammatory Diseases

Nice, France. 23-25 November 2011

Inflammatory mouse Ly6 $\mathrm{C}^{\text {high }}$ monocyte subset and its human counterpart, defined as $\mathrm{CD} 14^{+} \mathrm{CD} 16^{-}$, represent a valuable cellular target for innovative immunotherapeutic strategies against immune-mediated inflammatory disorders (IMID). However, delivery systems able to differentially target both subsets in vivo are still missing as well as demonstration for efficient immuno-modulation. The present work aims at providing evidences for the selective delivery of a siRNA-containing lipid formulation to the Ly- $6 C^{\text {high }}$ monocyte population and at evaluating the therapeutic potential of targeting this subset as well as their human counterpart for immuno-intervention in a prototype IMID like rheumatoid arthritis (RA). The preB-cell colony enhancing factor (PBEF/visfatin/Nampt) is an essential enzyme in the NAD biosynthetic pathway that exerts a key role in the persistence of inflammation through the induction of the expression of the TNF- $\alpha$ and IL- 6 pro-inflammatory cytokines and is highly expressed in patients with a variety of IMID. Mice with collagen-induced arthritis (CIA) display Ly-6C $\mathrm{C}^{\text {high }}$ monocytosis in the circulation that infiltrate into the inflamed joints. The systemic delivery of siRNAs formulated with the cationic liposome DMAPAP provides specific and functional down-regulation of PBEF within inflammatory monocytes. Moreover, decreased production of the PBEF-induced pro-inflammatory cytokines TNF- $\alpha$ and IL- 6 was evidenced in both mouse and human inflammatory monocytes. PBEF gene silencing within Ly- $6 C^{\text {high }}$ monocytes resulted in reduced disease severity in mice with CIA, associated with an overall systemic immunomodulation of the effector $\mathrm{T}$ cell balance. These results identify PBEF as a critical target to modulate autoimmune responses and inflammation in arthritis and provide novel evidence that silencing of a master gene within inflammatory monocytes is a promising strategy for future therapeutic intervention in the context of IMID.

\section{Author details}

'Inserm, U 844, CHU Saint Eloi University Hospital, Montpellier, France. ${ }^{2}$ University of Medicine, Montpellier, France. ${ }^{3}$ Inserm, U 1022, UFR des Sciences Pharmaceutiques et Biologiques, Paris, France. ${ }^{4} \mathrm{CNRS}$, UMR8151, Paris, France. ${ }^{5}$ University of Pharmacy Paris Descartes, Laboratoire de Pharmacologie Chimique, Génétique et Imagerie, Paris, France. ${ }^{6}$ Ecole Nationale Supérieure de Chimie de Paris, Paris, France. ${ }^{7} \mathrm{CHU}$ Lapeyronie University Hospital, Clinical Dept. for Osteoarticular Diseases, Montpellier, France. ${ }^{8}$ Center of Experimental Rheumatology, University Hospital Zurich and Zurich Center of Integrative Human Physiology, Zurich, Switzerland.

Published: 23 November 2011

doi:10.1186/1479-5876-9-S2-P38

Cite this article as: Présumey et al: Targeted delivery to inflammatory monocytes for efficient RNAi-mediated immuno-intervention in autoimmune arthritis. Journal of Translational Medicine 2011 9(Suppl 2):P38. 